\title{
Analysis of Molecular Interactions Using the Thermophoresis Method and its Applications in Neuroscience and Biological Processes
}

\author{
Sara Azizishalbaf ${ }^{1}$, Asadollah Asadi ${ }^{2 *}$, Arash Abdolmaleki ${ }^{3,4}$ \\ ${ }^{1}$ Basic Science Research Center, University of Tabriz, Tabriz, Iran \\ ${ }^{2}$ Department of Biology, Faculty of Sciences, University of Mohaghegh Ardabili, Ardabil, Iran \\ ${ }^{3}$ Department of Engineering Sciences, Faculty of Advanced Technologies, University of Mohaghegh Ardabili, Namin, Iran \\ ${ }^{4}$ Bio Science and Biotechnology Research center (BBRC), Sabalan University of Advanced Technologies (SUAT), Namin, Iran
}

\section{Article Info:}

\section{ABSTRACT}

Introduction: Molecular interactions play an important role in the phenomenon and biological processes. In fact, any cellular biological process ranged from genetic replication to the production of various proteins to the transmission of neurological, hormonal, membrane involves collections of molecular interactions that occur continuously. Interference in each of these processes at every stage of molecular interaction may be caused by various diseases. Therefore, their careful study can improve our understand of biological phenomena and lead to the emergence of new methods for the treatment of many diseases, in particular, the diseases of the nervous system. For this purpose, various biophysical and biochemical methods have been developed. Among them, infrared laser-based methods are very useful and important. Thermophoresis is one of these methods, which has been considered in recent years by medical, pharmaceutical, neuroscience, regeneration and biology scientists. This method is based on the direct movement of molecules in a temperature slope. This type of movement greatly depends on the biophysical properties of the molecule, including size, load, and solvent layer. Micro-scale modifications in this specification can affect the properties of the molecule under the influence of temperature. Conclusion: Thermophoresis is an easy, accurate, and fast method for analyzing the behavior of molecules. For this purpose, in the present study,

Key words:

1. Molecular Biology

2. Biological Phenomena 3. Nervous System Diseases we discussed the various theoretical and practical details as well as the various advantages and disadvantages of measuring the tendency of small molecules, such as ions and biochemical molecules.

*Corresponding Author: Asdollah Asadi

E-mail:asad.asady@gmail.com 


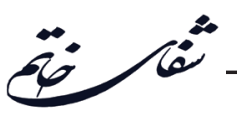

\title{
تجزيه و تحليل تعاملات مولكولى با استفاده از روش ترموفورز و كاربردهاى آن در علوم اعصاب و

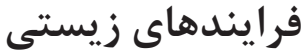

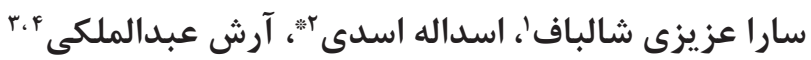

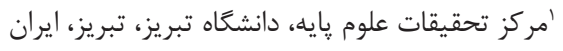

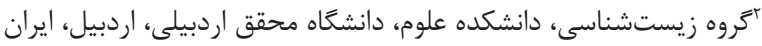

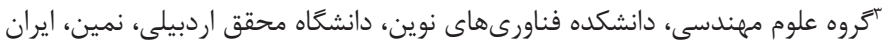

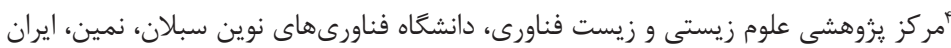

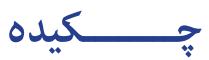

مقدمه: تعاملات مولكولى نقش مهمى را در يديدهها و فرايندهاى زيستى ايفاء مى كنند. درواقع هر فر فرايند

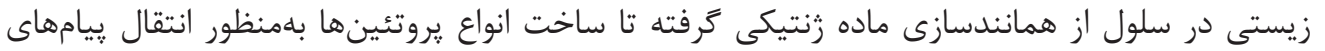

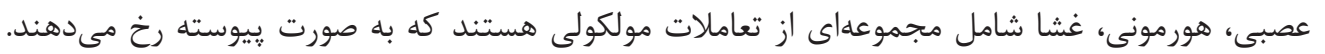

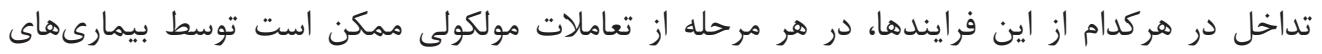

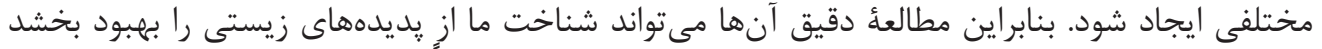

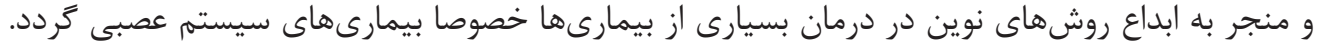

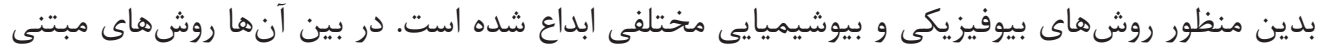

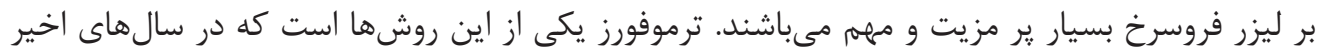

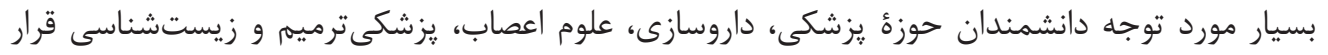

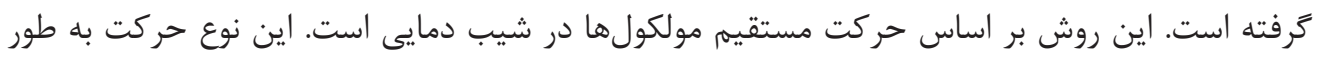

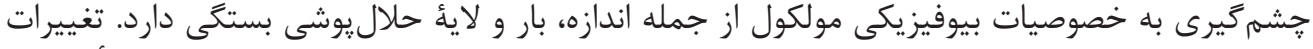

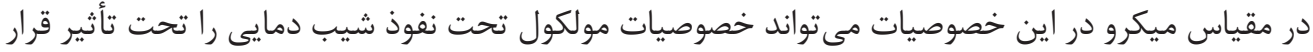

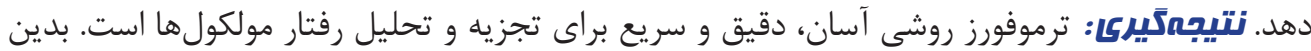

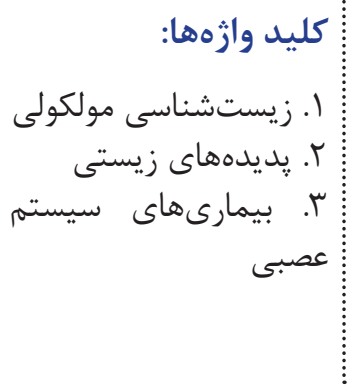

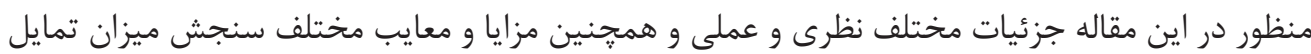

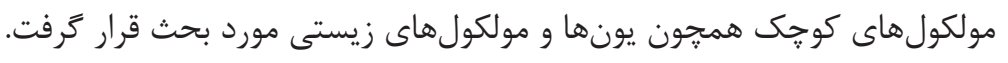




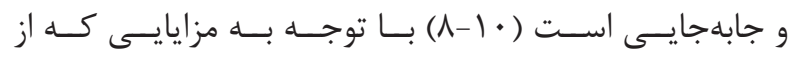

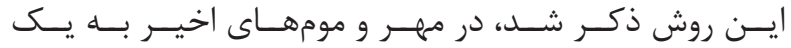

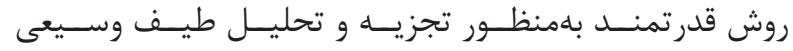

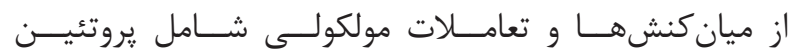

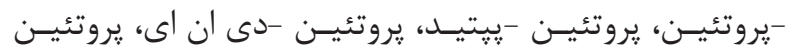

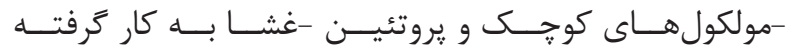

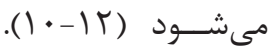

\section{ديدكاه نظرى ترموفورز}

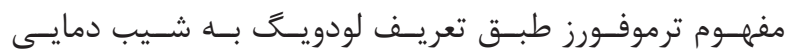

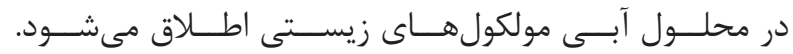

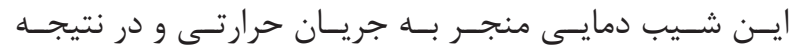

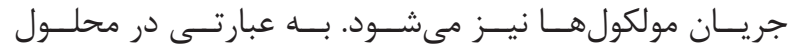

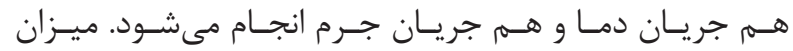

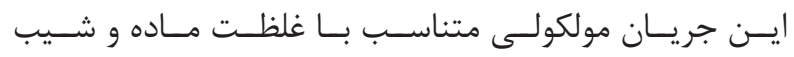

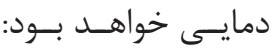

$$
j=-c D_{T} \operatorname{grad} T
$$

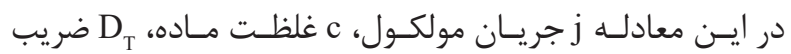

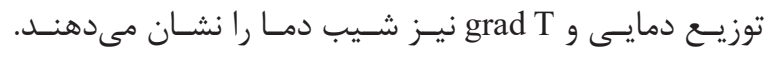
در حالـت كَذار steady state جريـان ترموفـورزى بــا جريـان

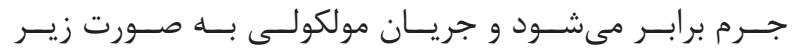

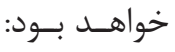

$$
j=-D \operatorname{grad} c
$$

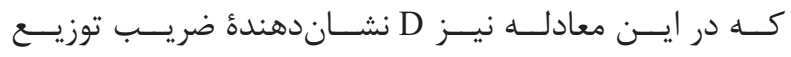
مى باشـــد.

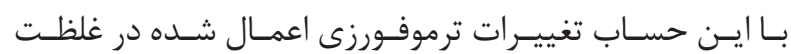

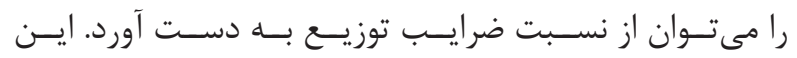

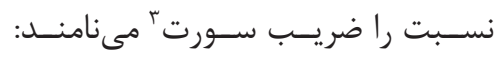

$$
S_{T}=\frac{D}{D_{T}}
$$

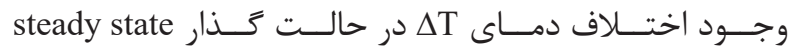

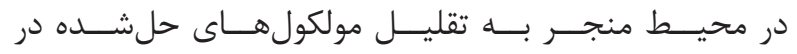

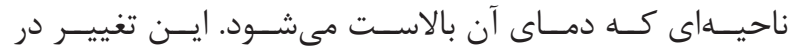

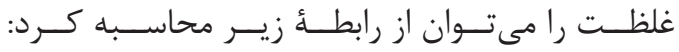

$$
C \text { L } C \text { L } / C \text { L }-\exp \left(-\mathrm{S}_{\mathrm{T}} \Delta \mathrm{T}\right)
$$

كـهـ C نشــانهُ غلظــت مولكولهـــا در نواحسى گَــرم و ســرد نمونـــه اسـت (9) نـانة (9) اجزا و نحوه كاركرد دستخاه

هـــر روش آزمايشـعاهى داراى اجـزا و تجهيزاتـى اسـت كـهـ

${ }^{1}$ Microscale thermophoresis

${ }^{2}$ Ludwig

${ }^{3}$ Soret 


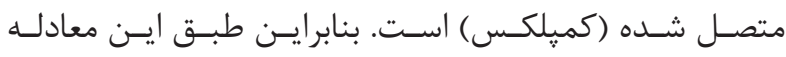

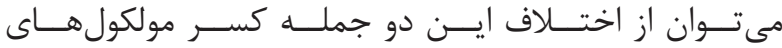

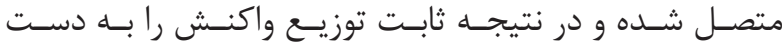

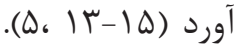

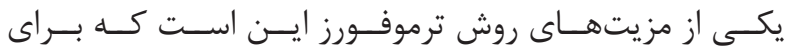

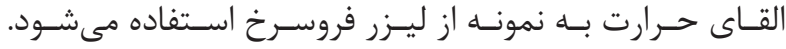

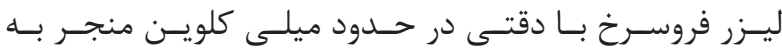

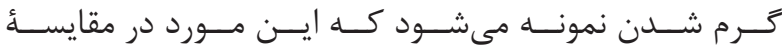

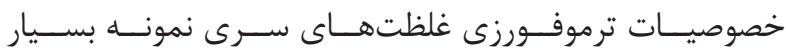

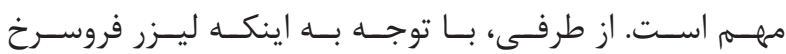

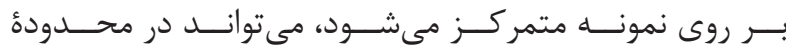

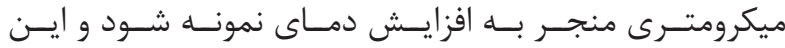

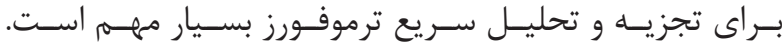

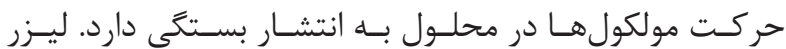

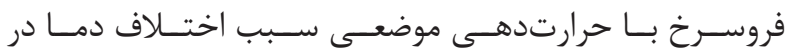

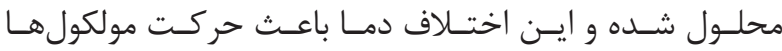

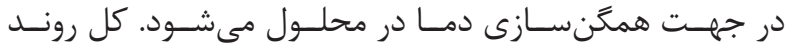

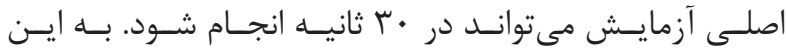

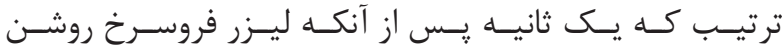

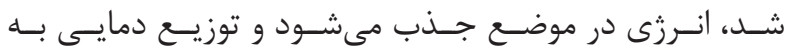

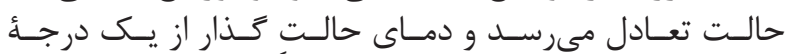

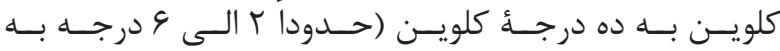

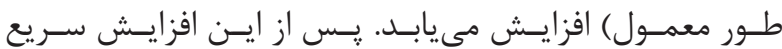

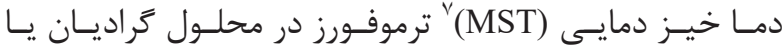

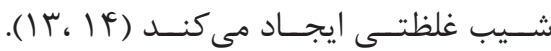

$$
\text { تفسير سيخنال هاب التها }
$$

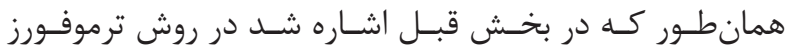

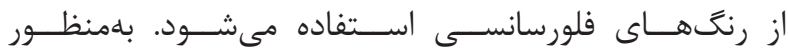

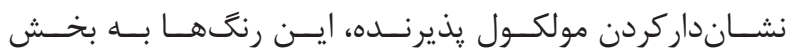

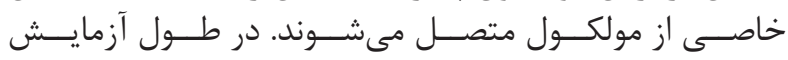

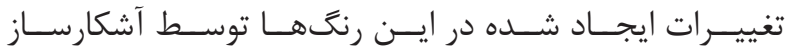

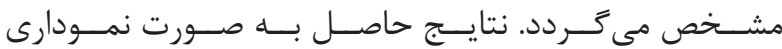

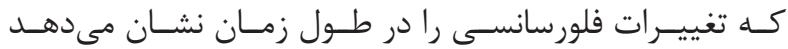

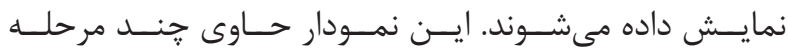

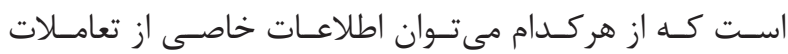

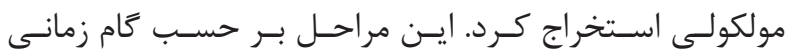

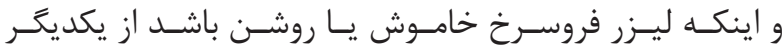

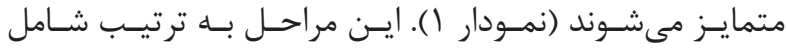

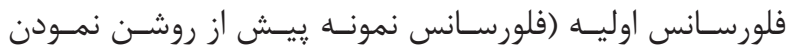

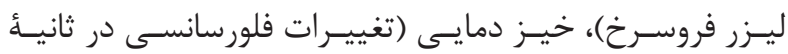

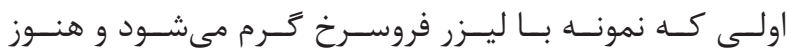

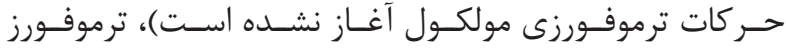

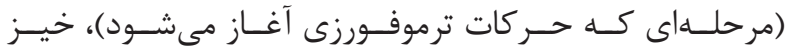

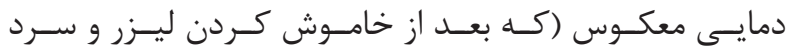

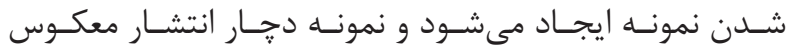

\footnotetext{
${ }^{4}$ Dichroic

${ }^{5}$ Capillary
}

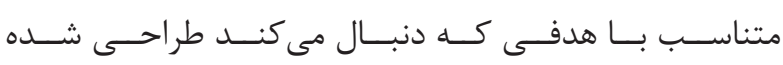

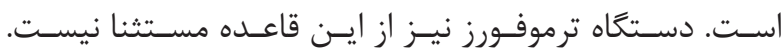

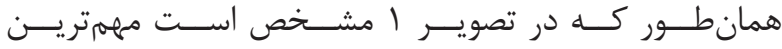

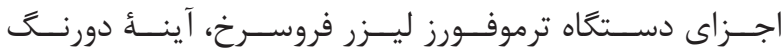

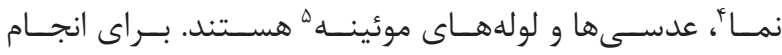

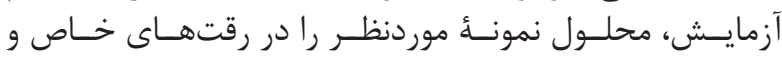

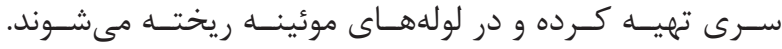

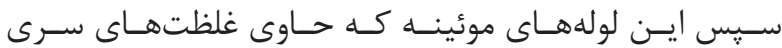

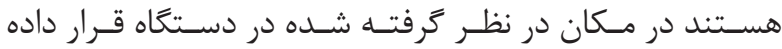

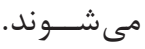

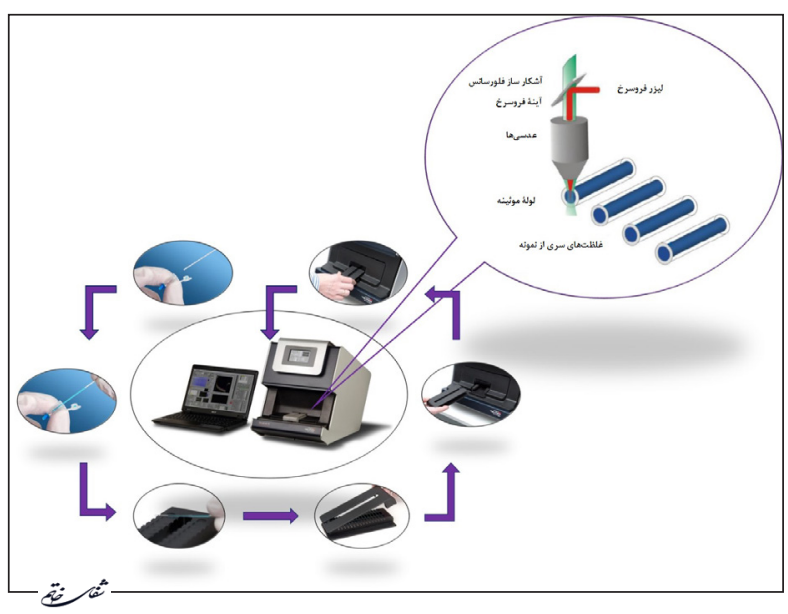

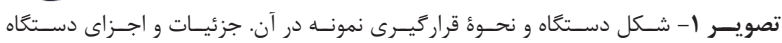

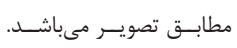

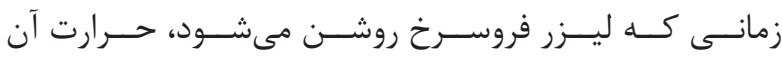

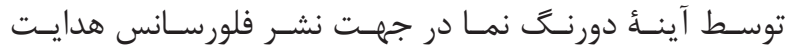

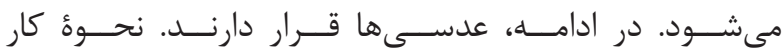

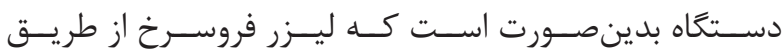

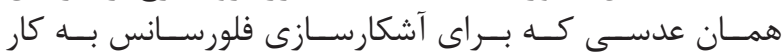

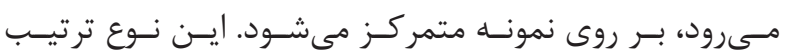

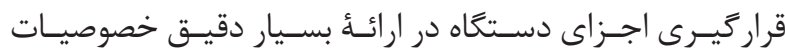

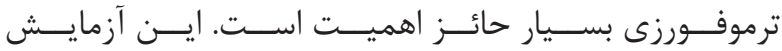

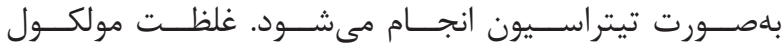

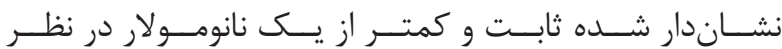

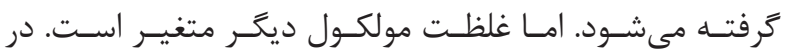

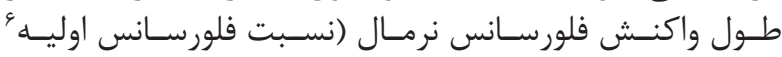

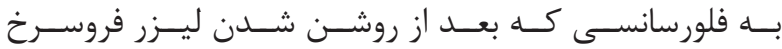

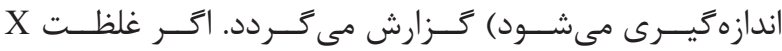

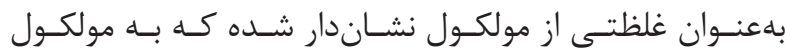

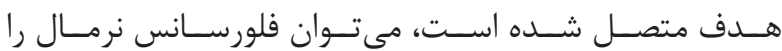

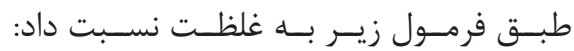

Fnorm 5(1 - x) Fnorm(unbound) +x Fnorm(bound)

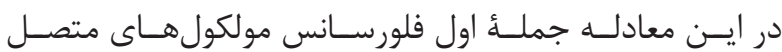

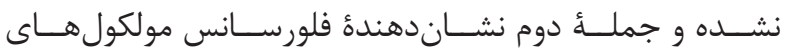

\footnotetext{
${ }^{6}$ Initial fluorescence

${ }^{7}$ MST T-jump
} 
خيز دمايى

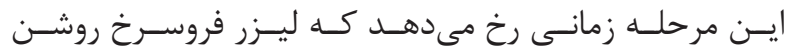

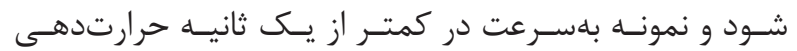

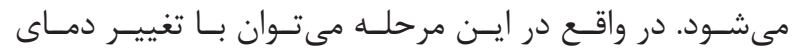

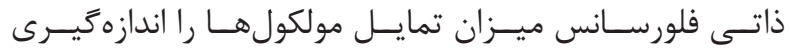

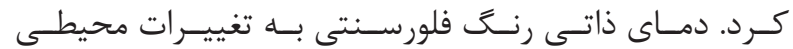

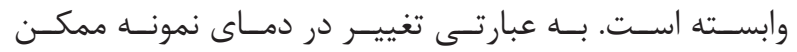

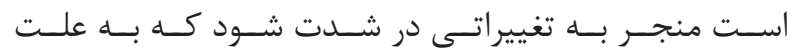

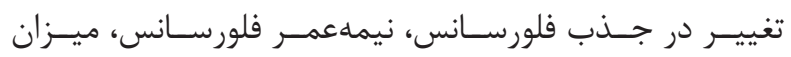

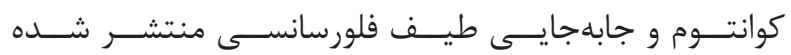

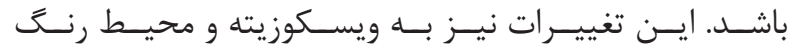

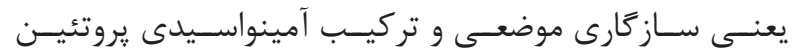

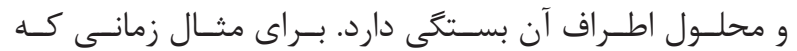

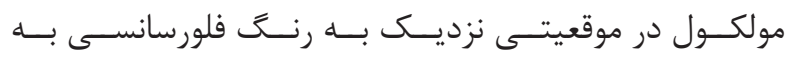

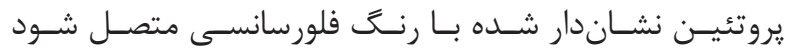

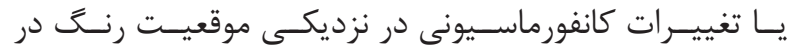

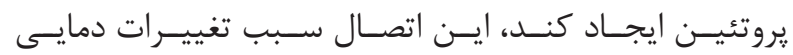

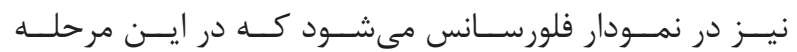

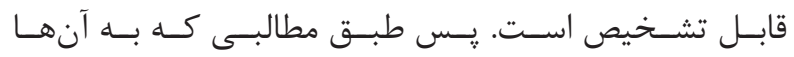

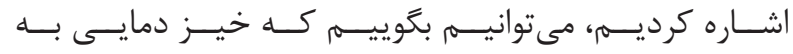

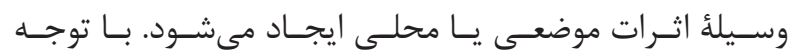

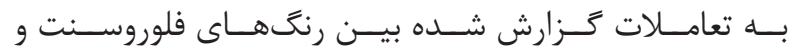

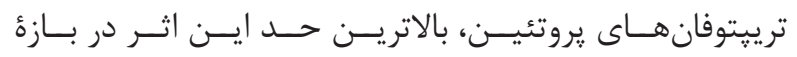

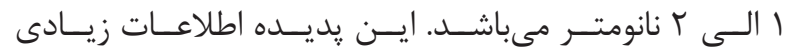

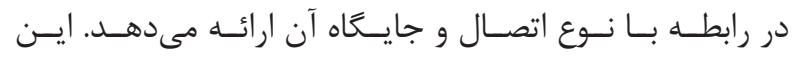

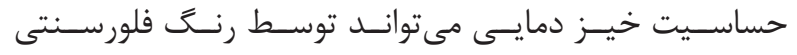

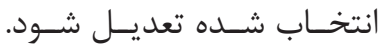

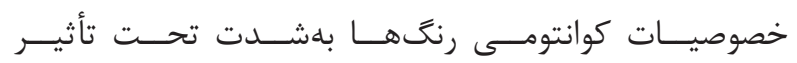

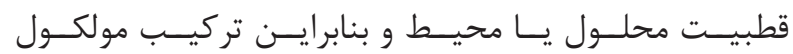

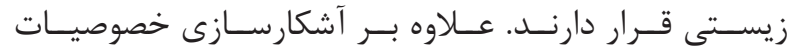

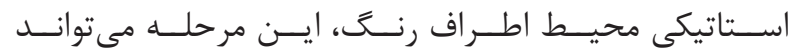

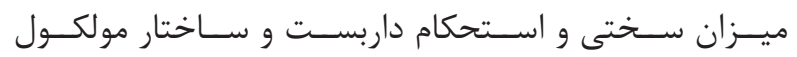
ديبولهــا را نيـز

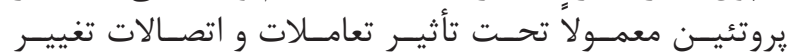

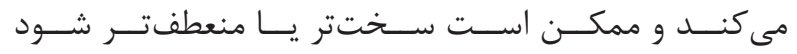

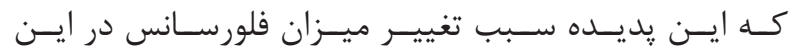

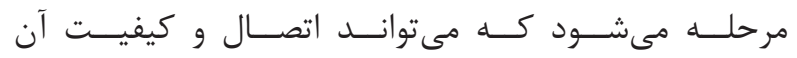

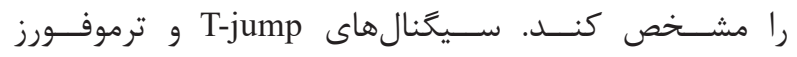

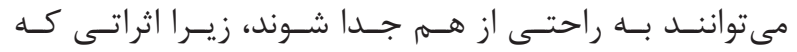

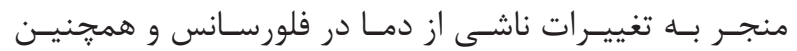

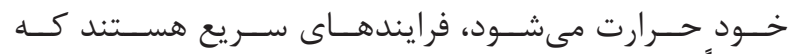

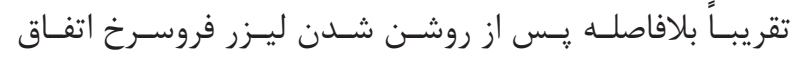

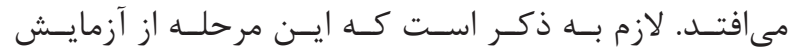

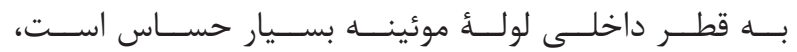

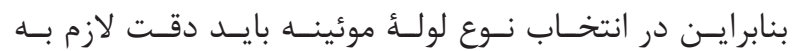
كار كرفتـهـ شـود (19 ، 1\% ، 19).

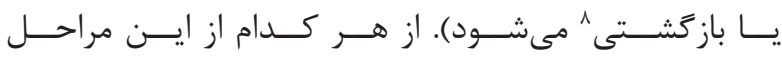

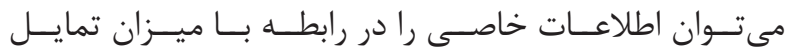

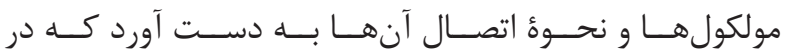

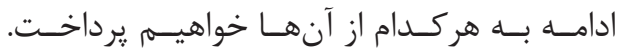

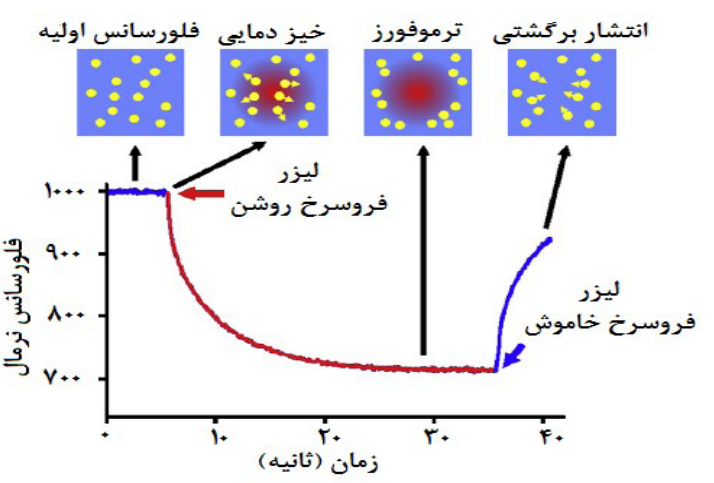

运

نمودار ا- سيكنال حاصل از آزمايش ترموفورز و مراحل مختلف آن.

فلورسانس اوليه

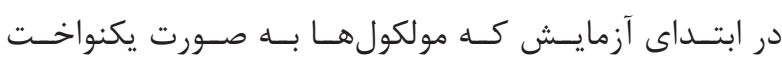

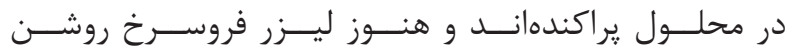

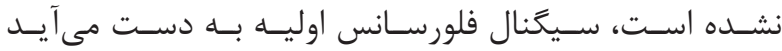

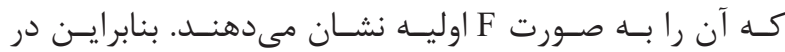

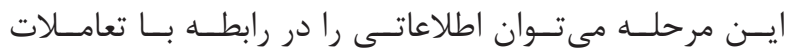

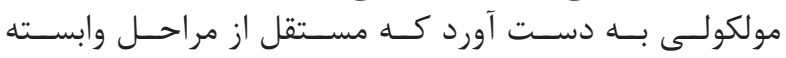

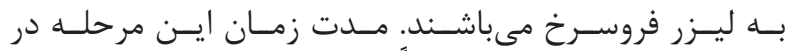

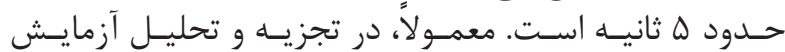

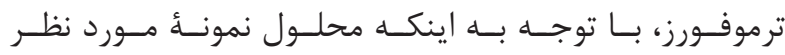

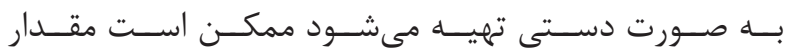

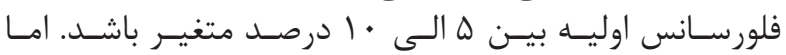

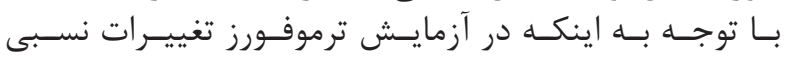

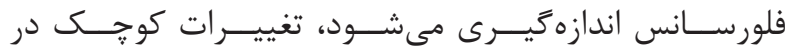

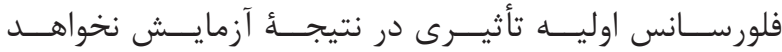

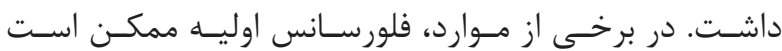

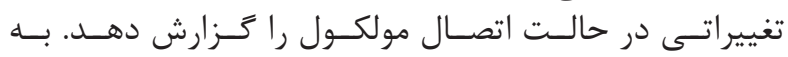

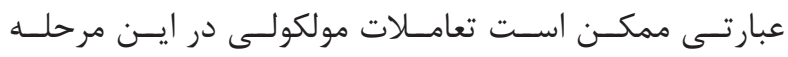

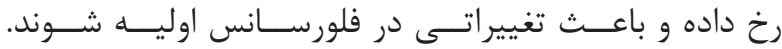

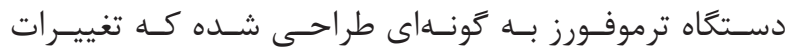

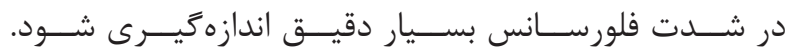

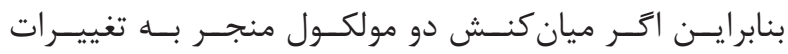

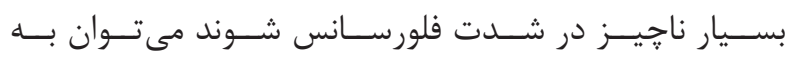

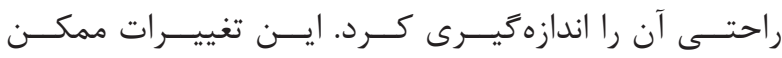

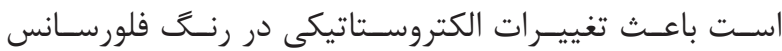

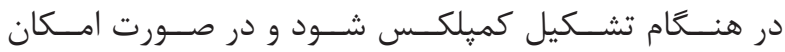

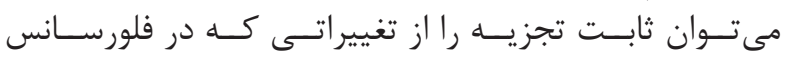

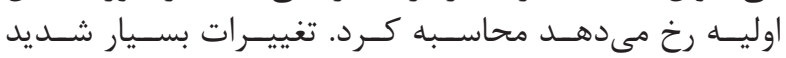

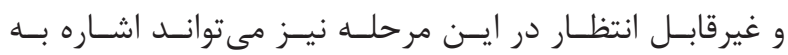

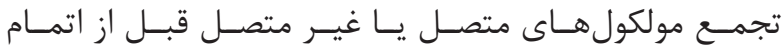

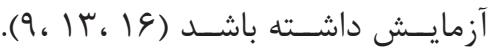

${ }^{8}$ Back diffusion 


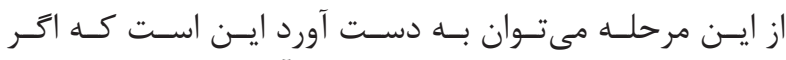

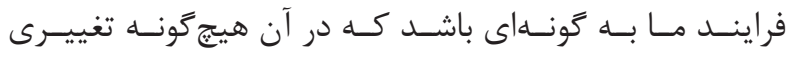

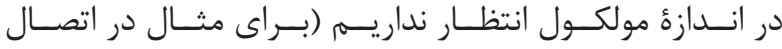

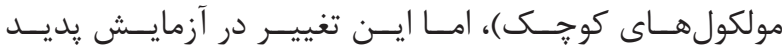

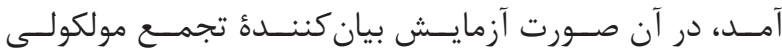

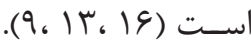

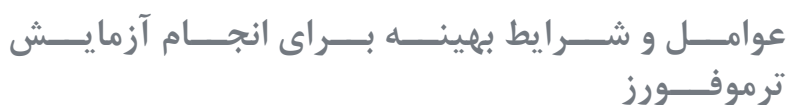

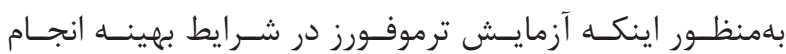

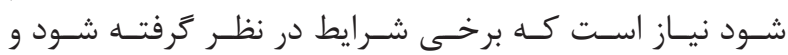

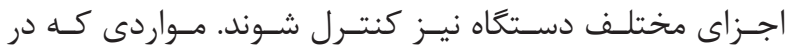

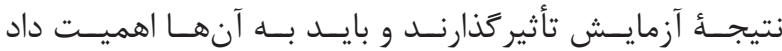

عبارتانســـ ازئ

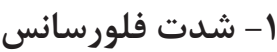

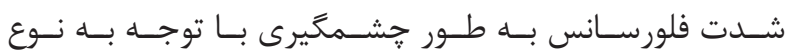

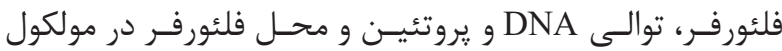

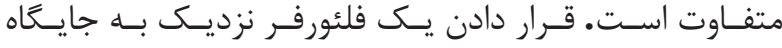

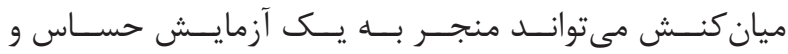

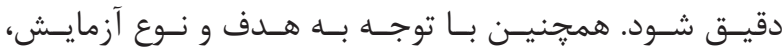

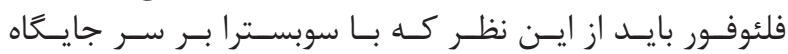

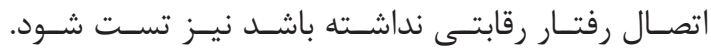

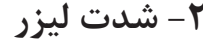

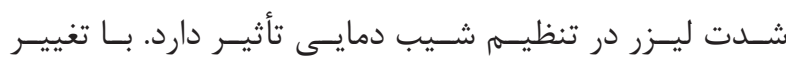

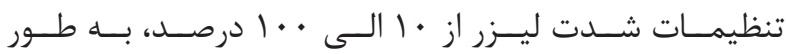

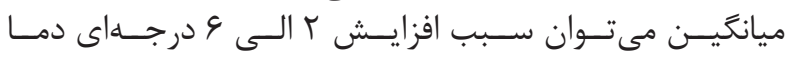

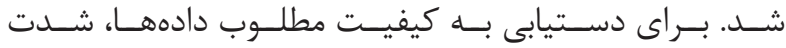

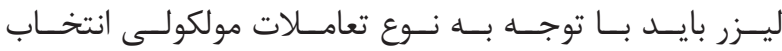

شــود.

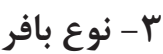

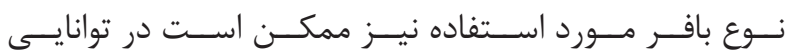

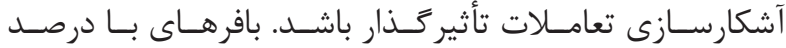

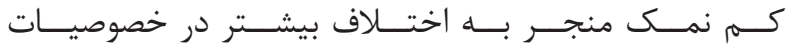

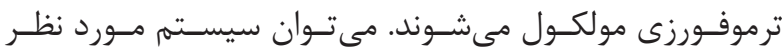

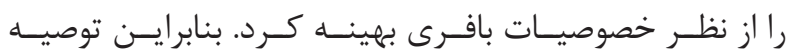

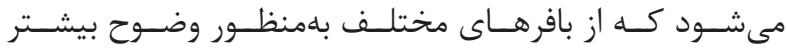

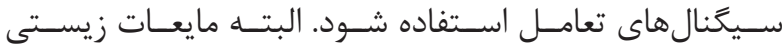

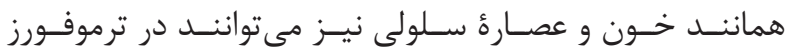

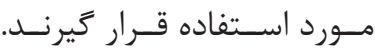

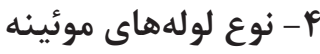

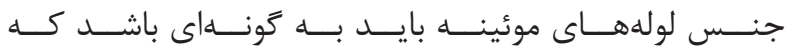

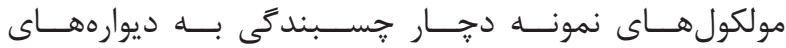

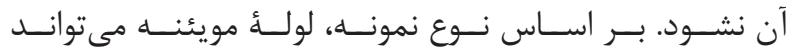

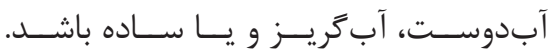

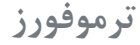

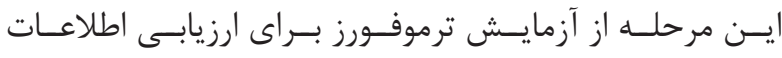

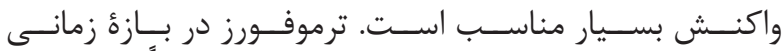

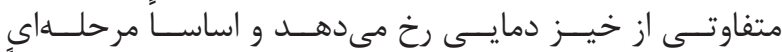

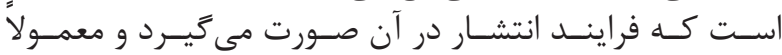

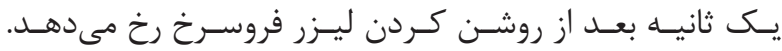

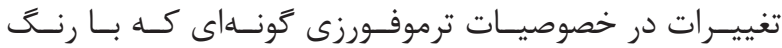

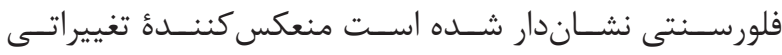

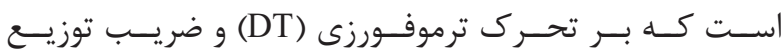

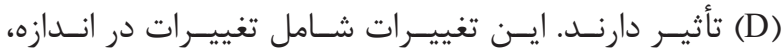

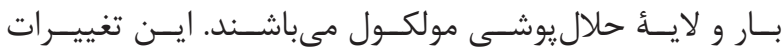

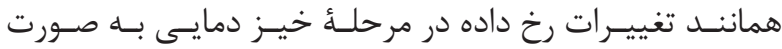

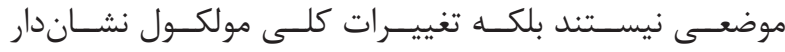

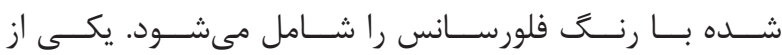

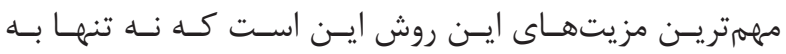

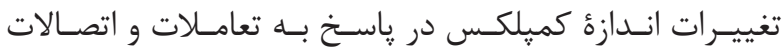

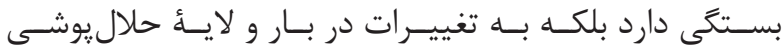

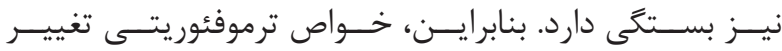

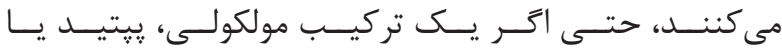

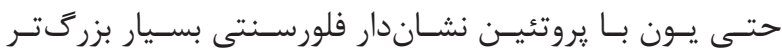

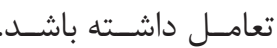

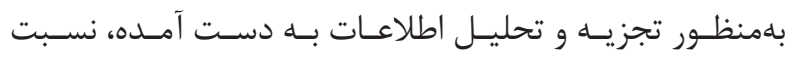

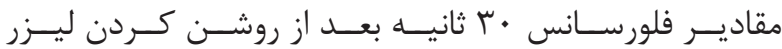

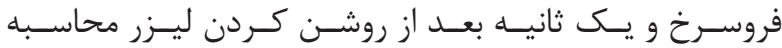

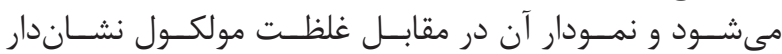

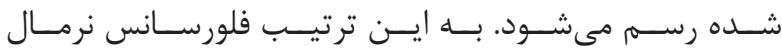

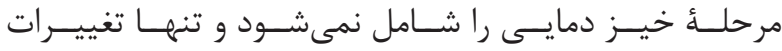

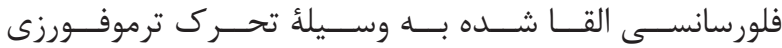

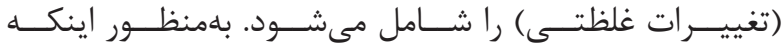

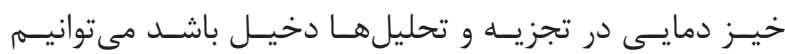

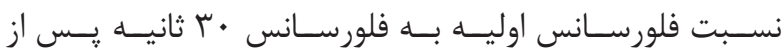

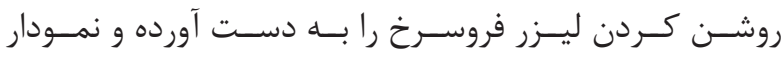

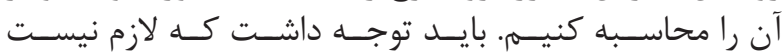

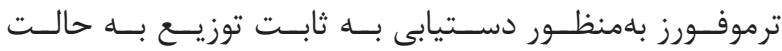

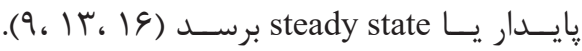

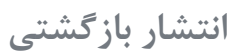

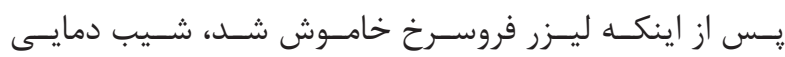

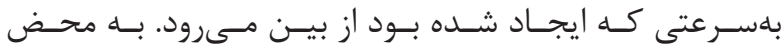

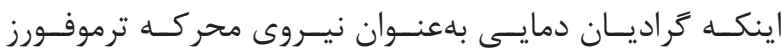

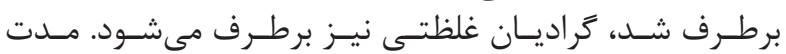

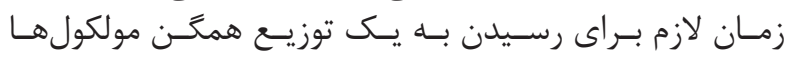

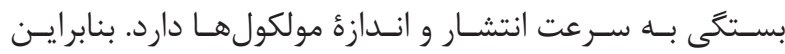

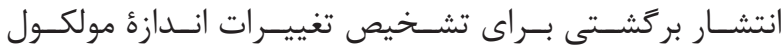

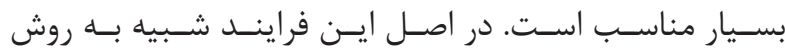

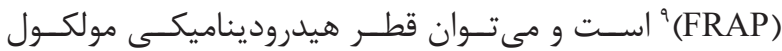

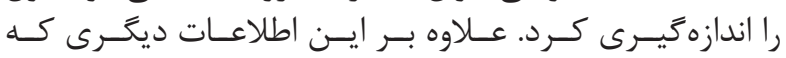

${ }^{9}$ Fluorescence recovery after photo bleaching 
شـده اسـت. بـا اتصـال كامـل توالى FV Fل آمينواسـيدى متصـل

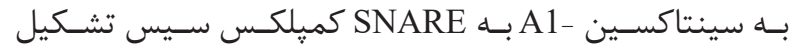

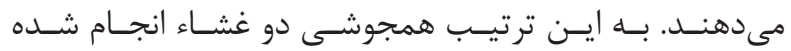

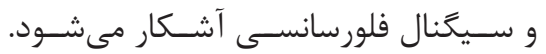

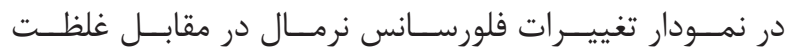

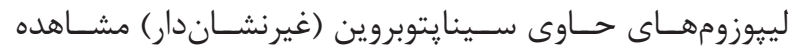

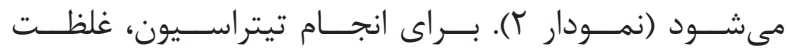

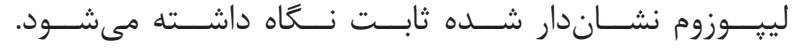

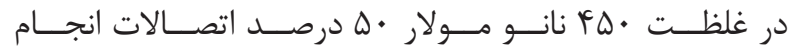

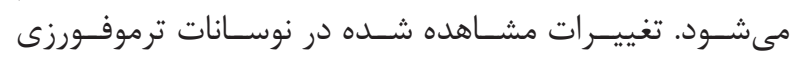

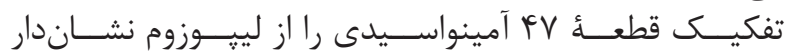

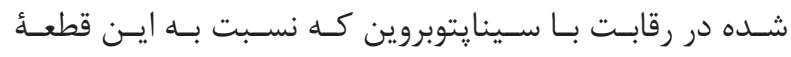

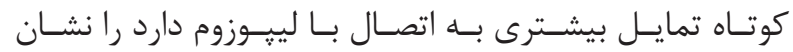

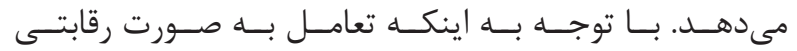

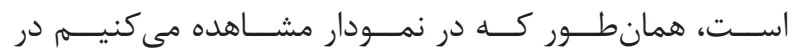

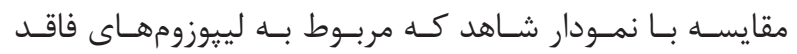

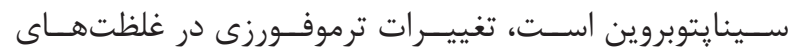

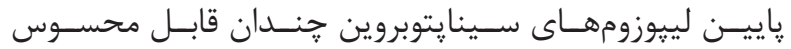

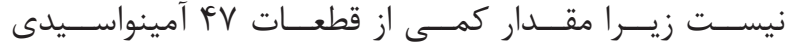

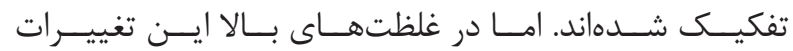
بسـيار قـوى و مشــهود اسـت (1) ، 11 ).
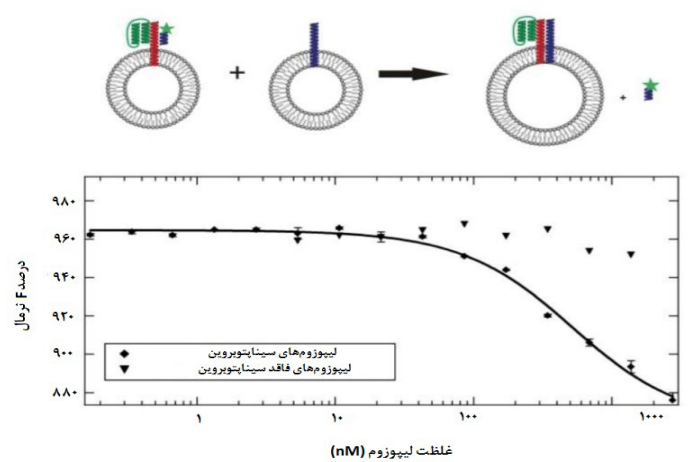

ثمث

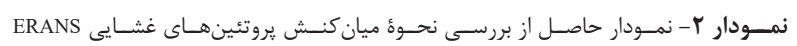
بــه وسـيلئ ترموفـورز.

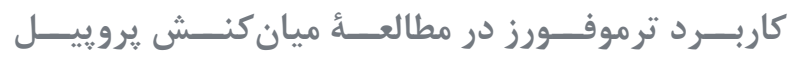

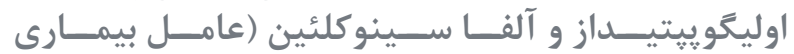

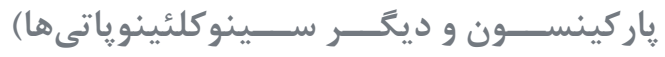

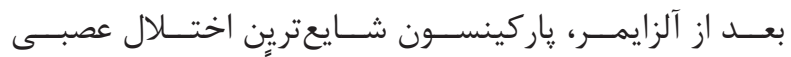

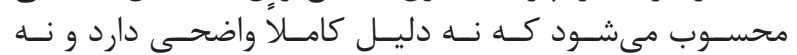

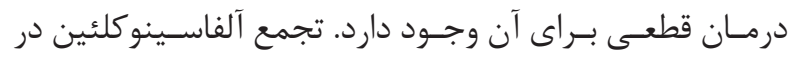

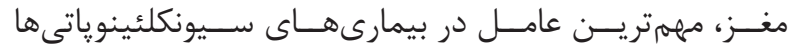

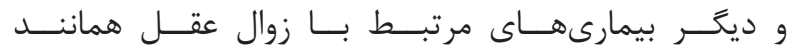

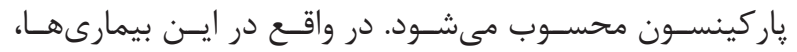

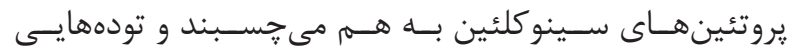

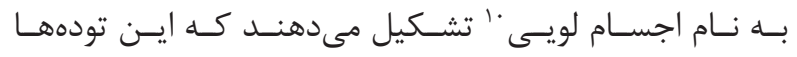

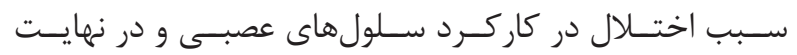

${ }^{10}$ Lewy bodies

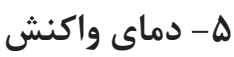

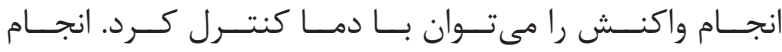

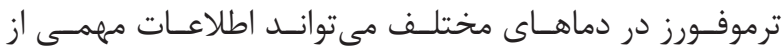

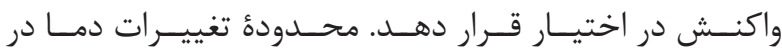

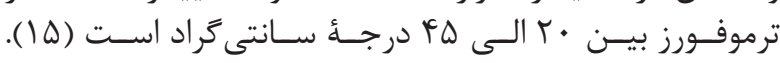
كسترة كاربردى روش ترموفورز

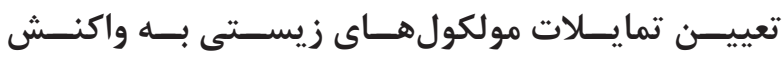

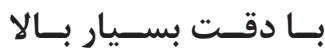

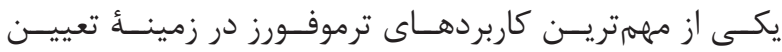

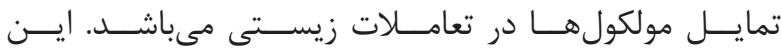

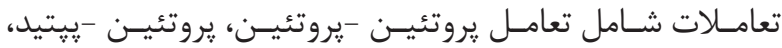

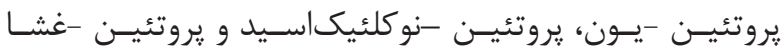

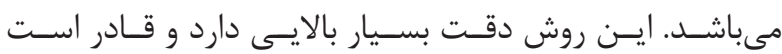

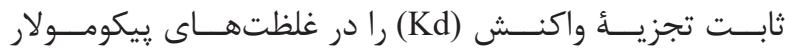

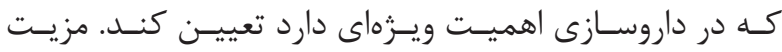

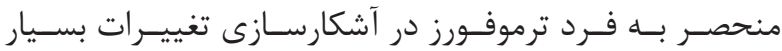

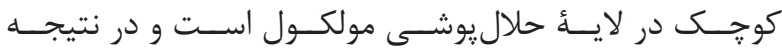

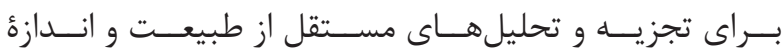

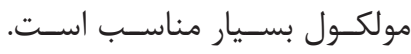

\section{ميان كنش يرو تئينهاى غشايى}

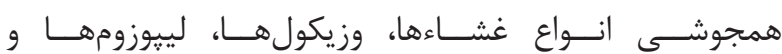

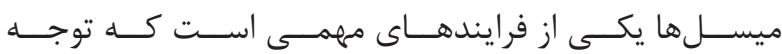

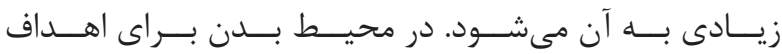

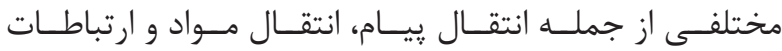

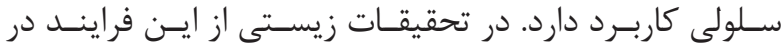

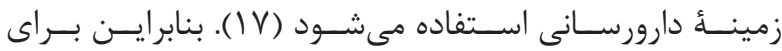

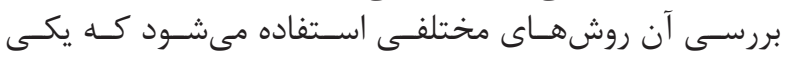

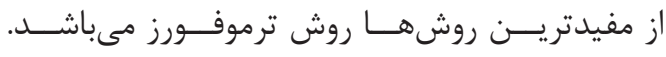

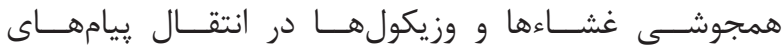

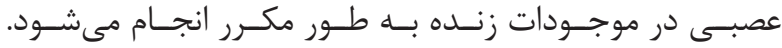

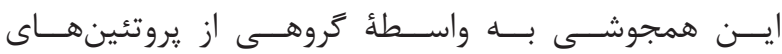

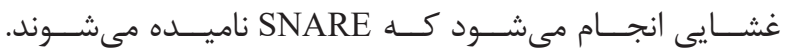

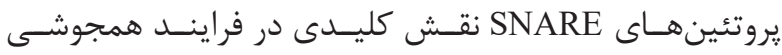

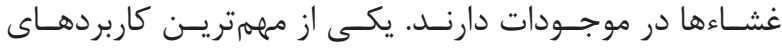

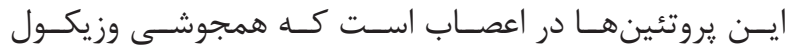

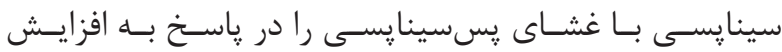

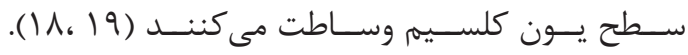

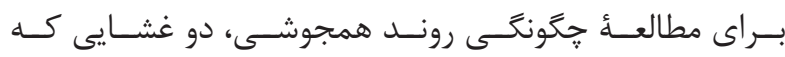

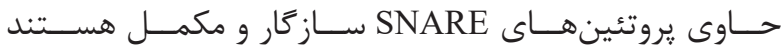

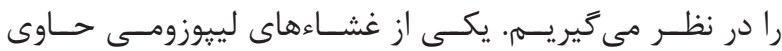

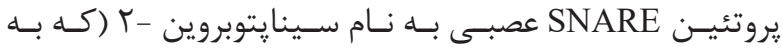

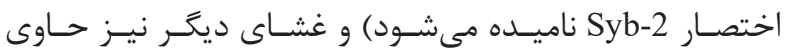

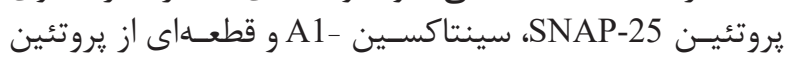

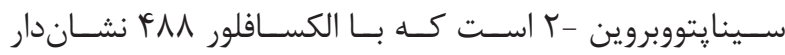




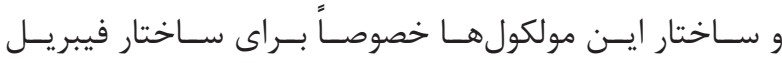
آلفاسـينوكلئين متفــاوت اســـــا

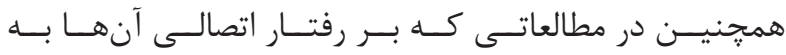

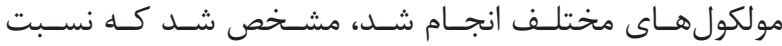

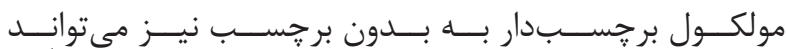

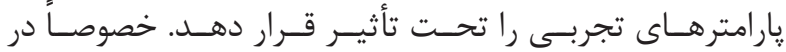

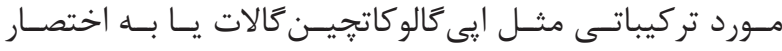

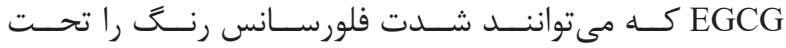

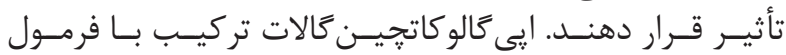

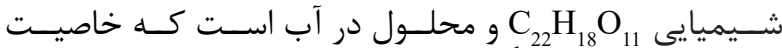

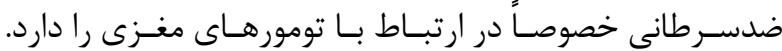

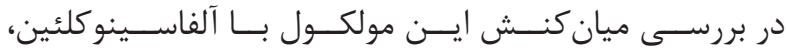

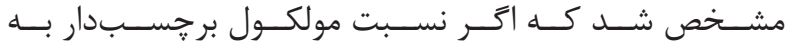

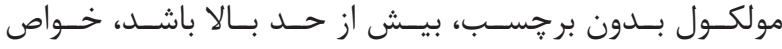

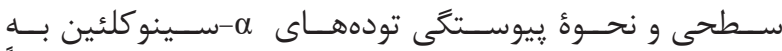

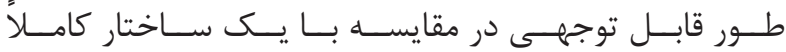

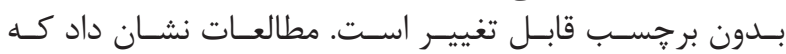

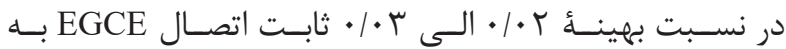

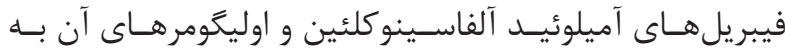

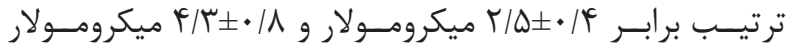

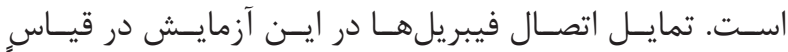

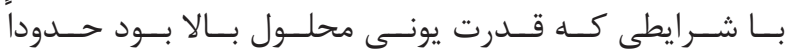

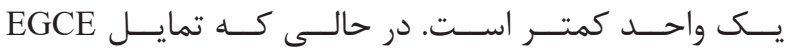

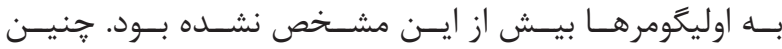

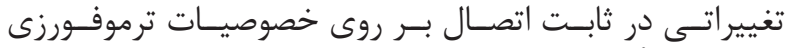

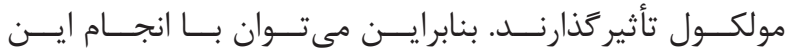

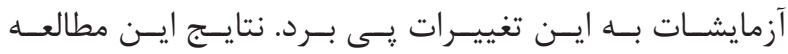

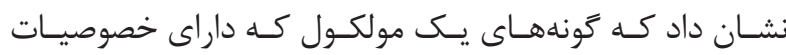

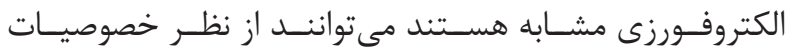

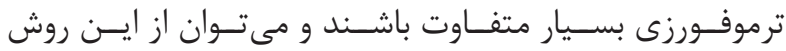

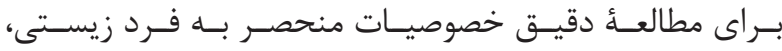

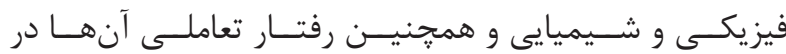

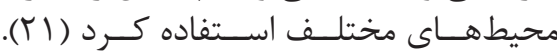

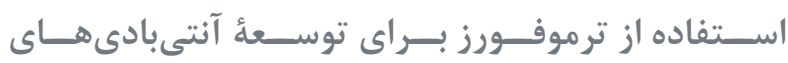

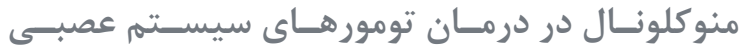

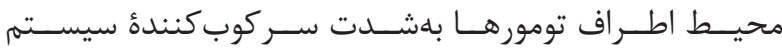

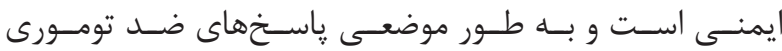

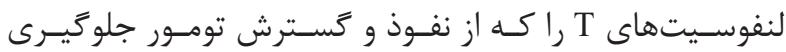

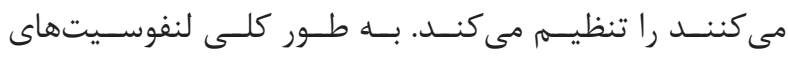

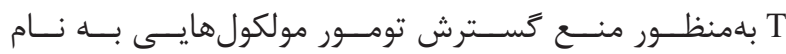

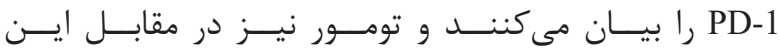

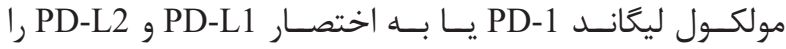

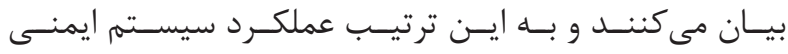

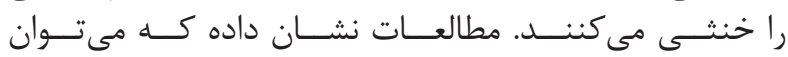

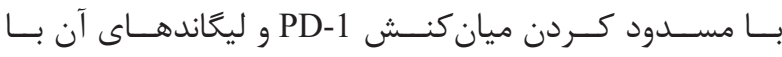

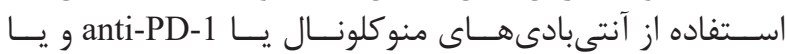

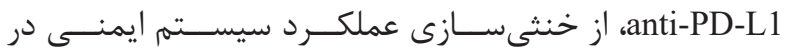

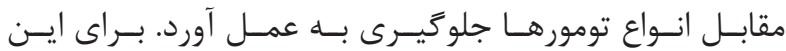

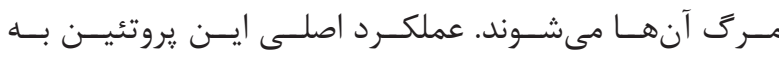

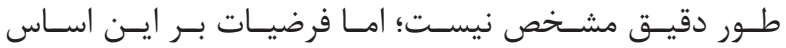

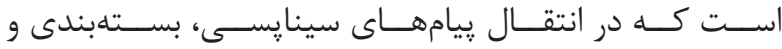

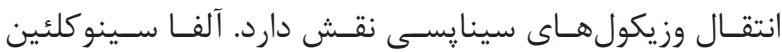

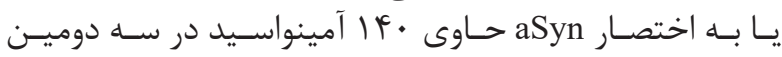

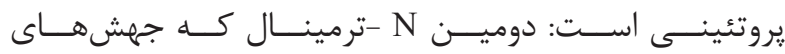

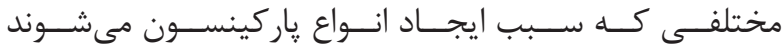

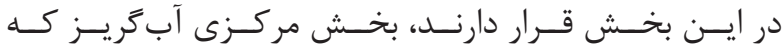

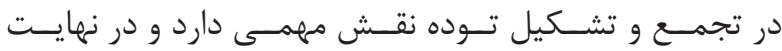

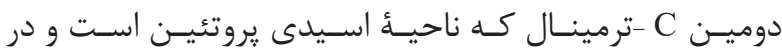

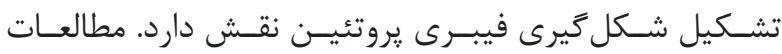

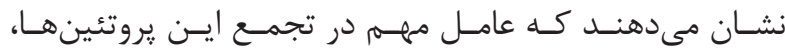

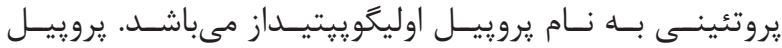

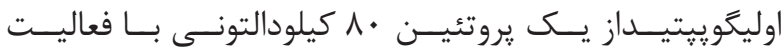

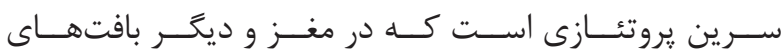

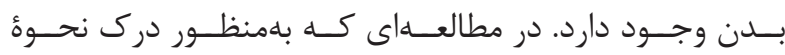

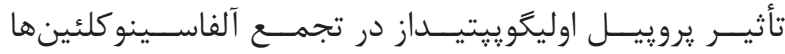

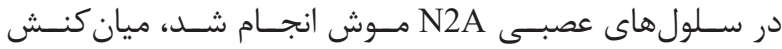

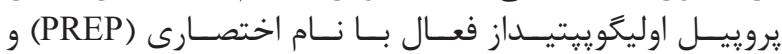

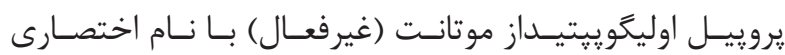

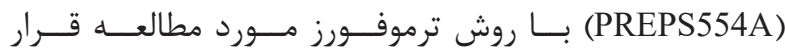

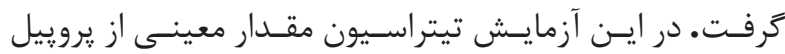

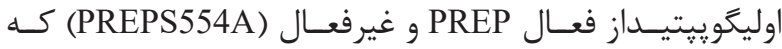

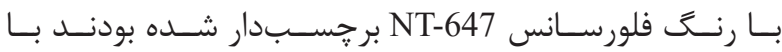

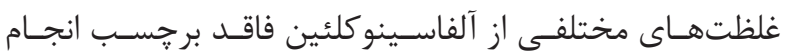

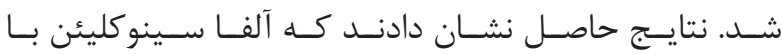

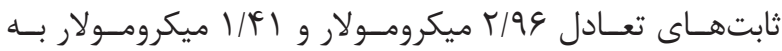

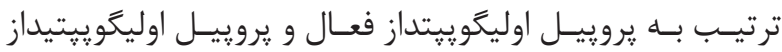

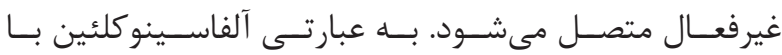

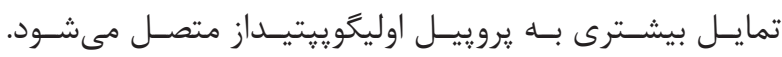

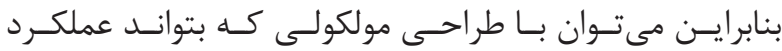

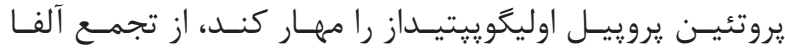

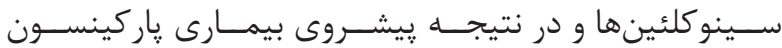

جلوكيـرى كـرد (•r) (r).

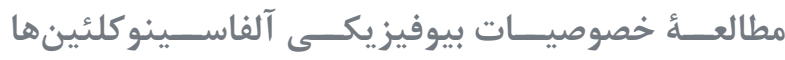

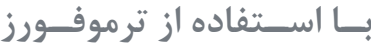

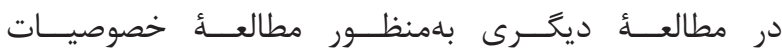

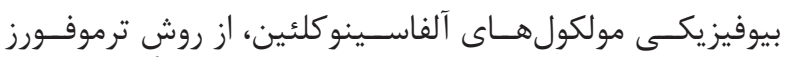

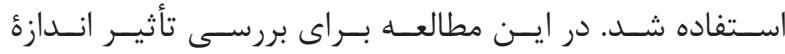

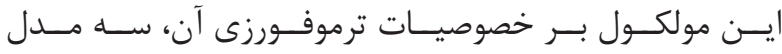

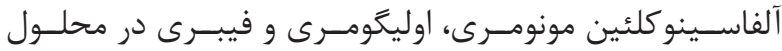

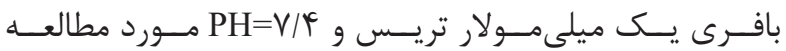

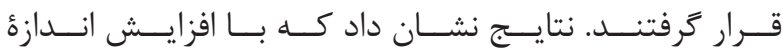

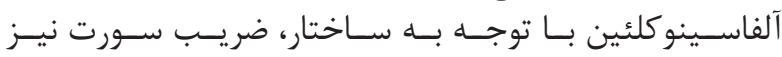

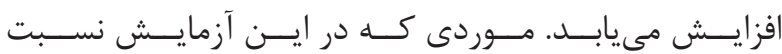

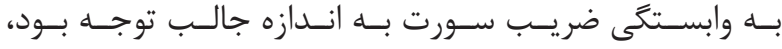

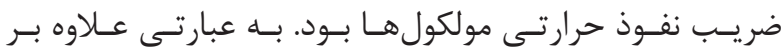

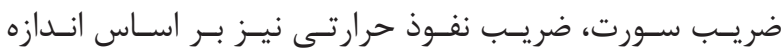




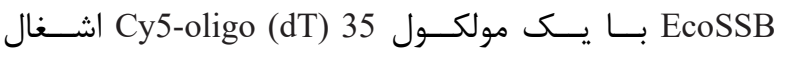

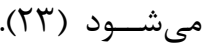

مطالعة سينتيك آنزيمها

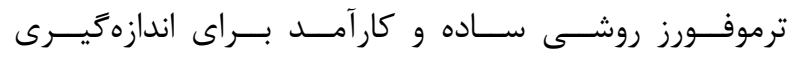

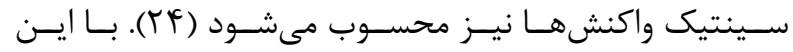

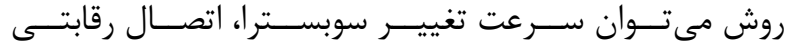

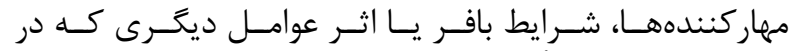

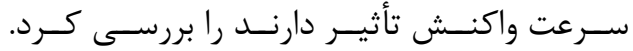

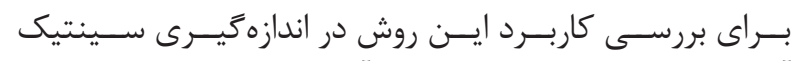

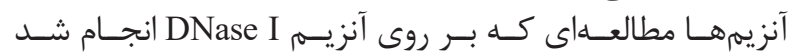

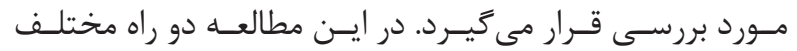

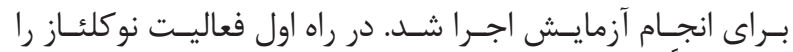

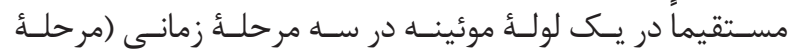

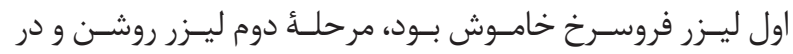

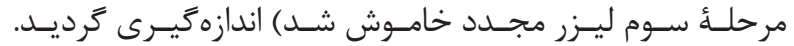

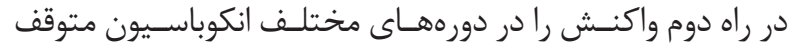

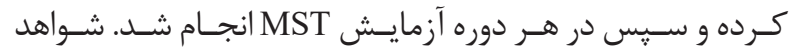

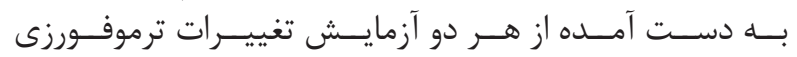

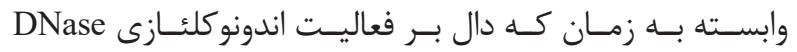

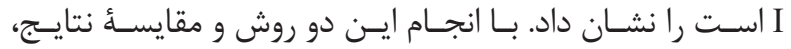

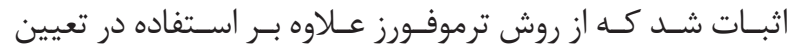

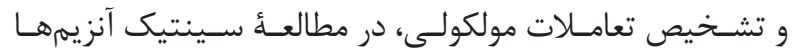

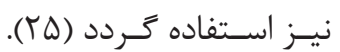

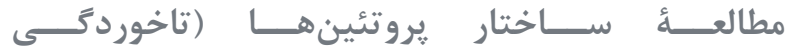

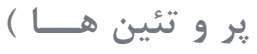

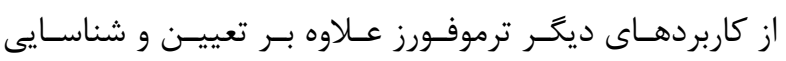

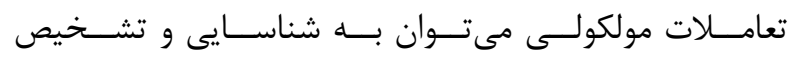

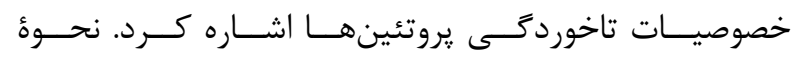

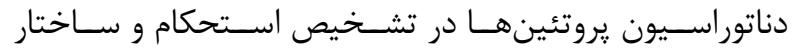

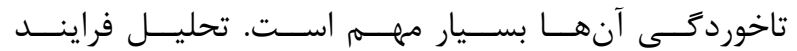

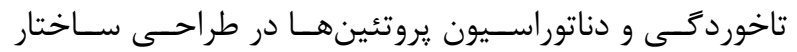

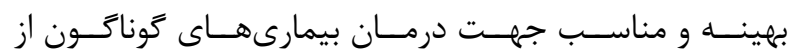

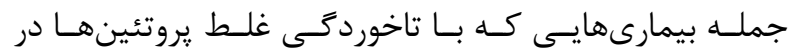

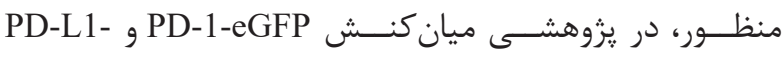

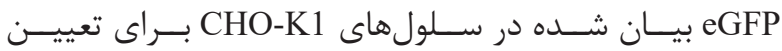

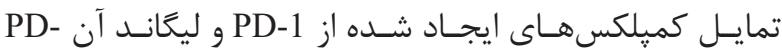

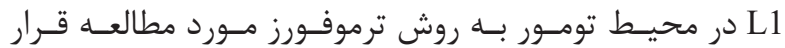

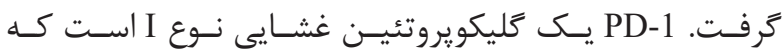

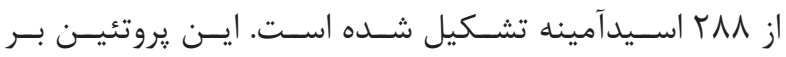

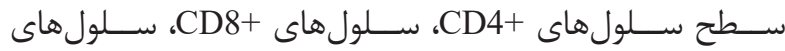

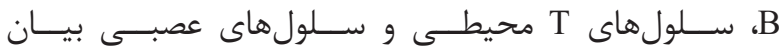

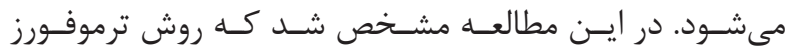

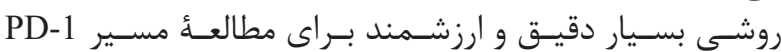
/ PD-L1

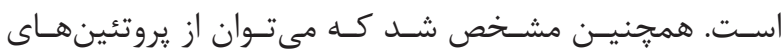

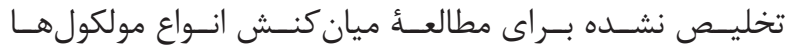

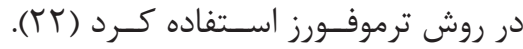

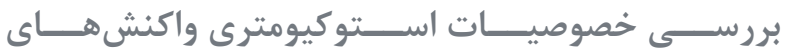
زيســتـى

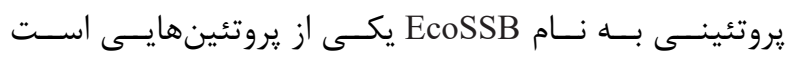

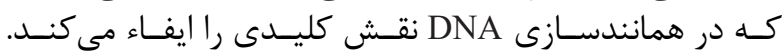

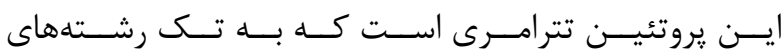

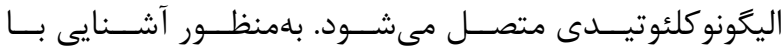

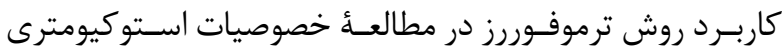

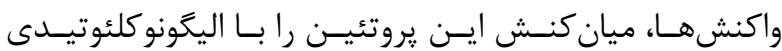

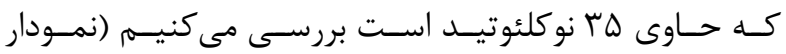

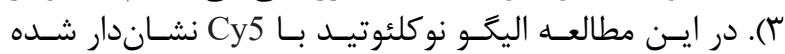

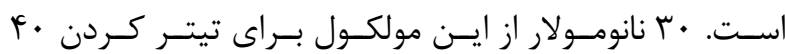

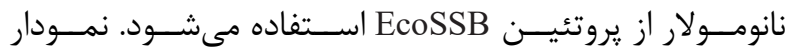

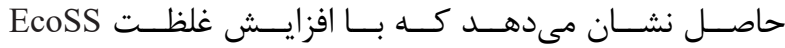

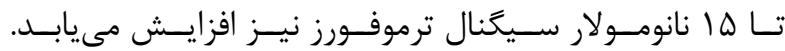

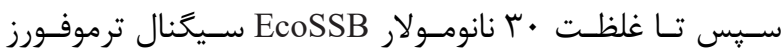

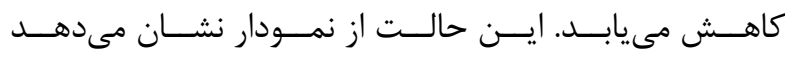

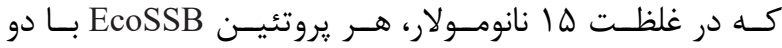

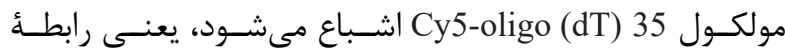

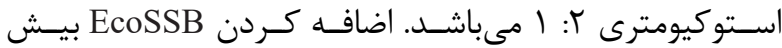

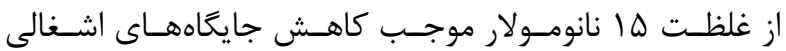

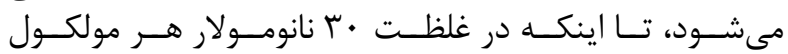

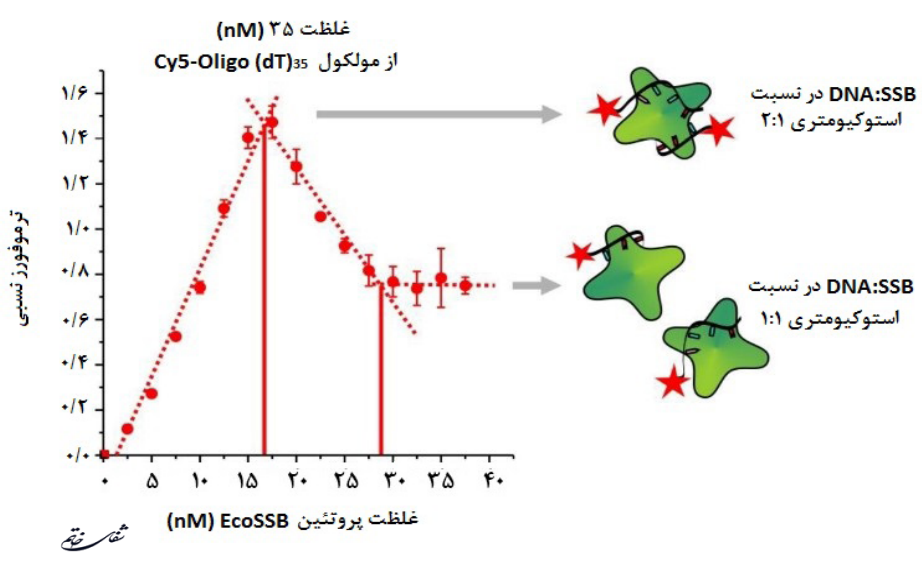

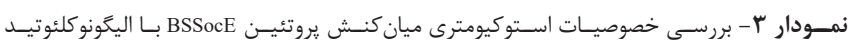




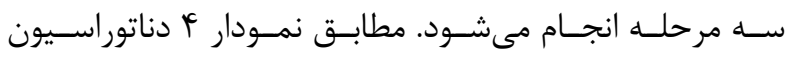

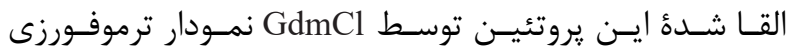

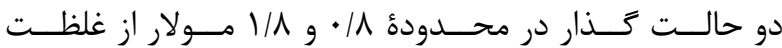
نشــان مى GdmCl

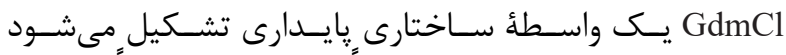

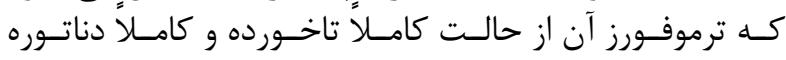

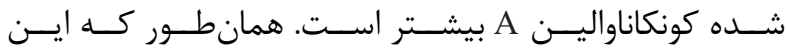

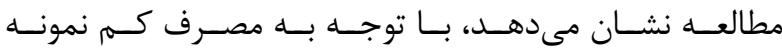

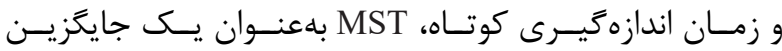

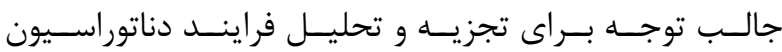

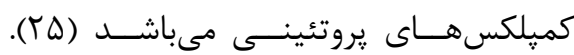
نتيجه كَيرى

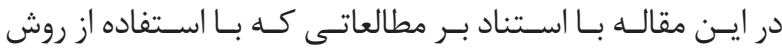

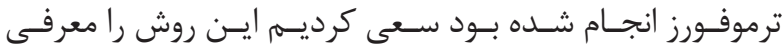

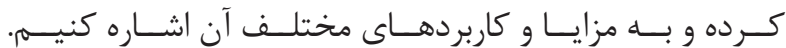

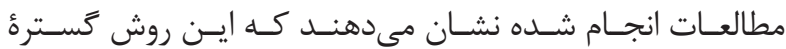

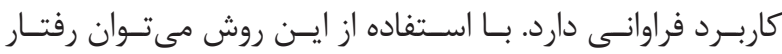

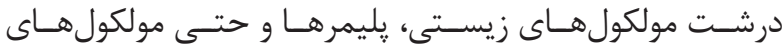

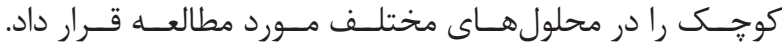

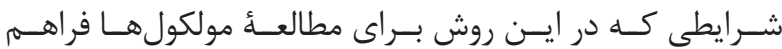

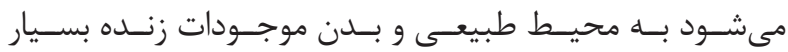

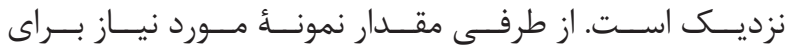

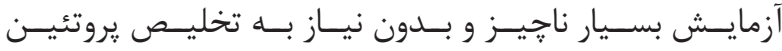

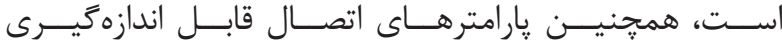

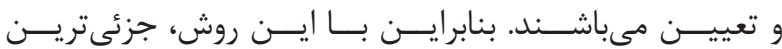

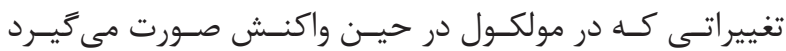

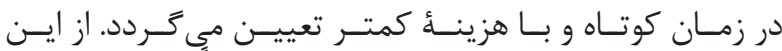

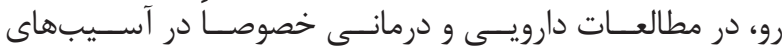

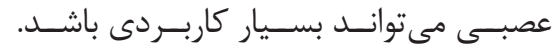

1. Fekri R, Salehi M, Asadi A, Kubicki M. Spectroscopic studies, structural characterization and electrochemical studies of two cobalt (III) complexes with tridentate hydrazone Schiff base ligands: Evaluation of antibacterial activities, DNA-binding, BSA interaction and molecular docking. Applied Organometallic Chemistry. 2018; 32(2): e4019.

2. Miri V, Asadi A, Mansourizadeh F, Sagha M, Ghasem Golmohammadi M. Fabrication and evaluation of the morphology, biodegradability, and chemical characteristics of the nano-fibrous scaffold poly-1lactic-acid (plla) and its application in neural tissue engineering. Journal of Urmia University of Medical Sciences. 2015; 25(11): 988-97.

3. Seidel SA, Wienken CJ, Geissler S, JerabekWillemsen M, Duhr S, Reiter A, et al. Label-free microscale thermophoresis discriminates sites and affinity of protein-ligand binding. Angew Chem Int Ed

$$
\text { ارتبــاط اســت بســيار حائـز اهميــت مىباشــد. }
$$

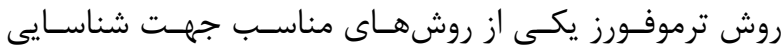

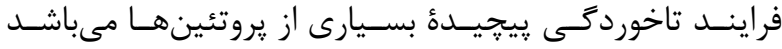

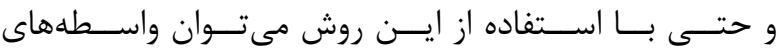

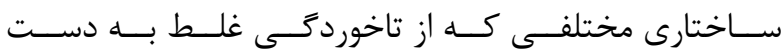

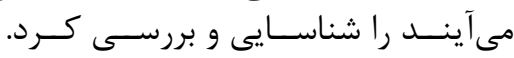

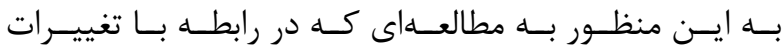

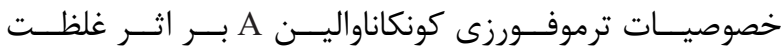
مdmCl

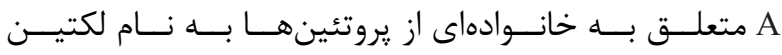

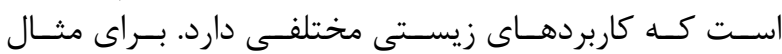

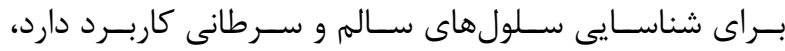

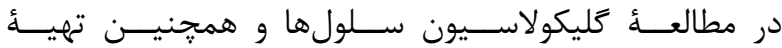

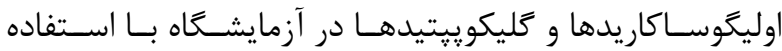

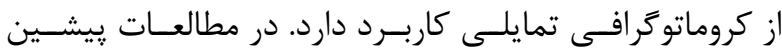

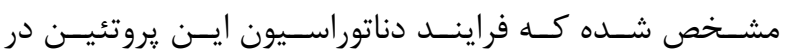

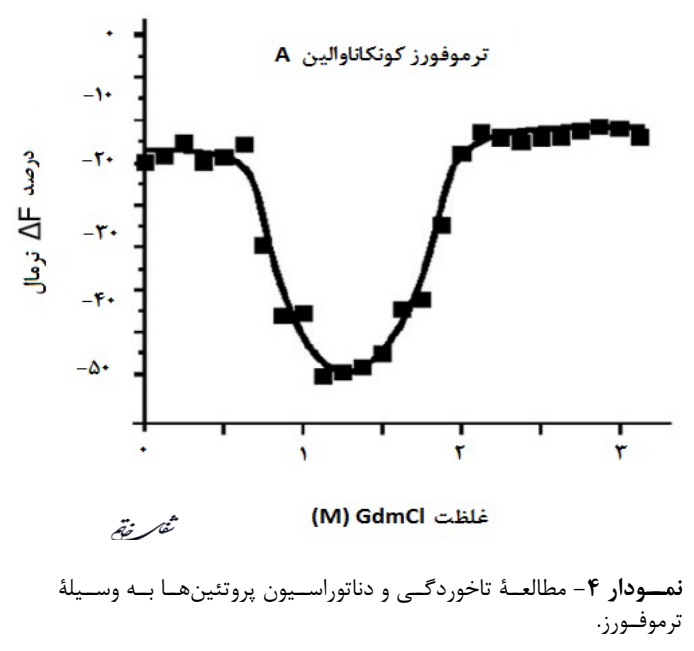

منابع

Engl. 2012; 51(42): 10656-9.

4. Bao J, Krylova SM, Wilson DJ, Reinstein O, Johnson PE, Krylov SN. Kinetic capillary electrophoresis with mass-spectrometry detection (kce-ms) facilitates labelfree solution-based kinetic analysis of protein-small molecule binding. Chembiochem. 2011; 12(17): 2551-4.

5. Breitsprecher D, Schlinck N, Witte D, Duhr S, Baaske $\mathrm{P}$, Schubert T. Aptamer binding studies using microscale thermophoresis. Methods Mol Biol. 2016; 1380: 99-111.

6. Piazza R. Thermophoresis: moving particles with thermal gradients. Soft Matter. 2008; 4(9): 1740-4

7. Entzian C, Schubert T. Studying small moleculeaptamer interactions using MicroScale Thermophoresis (MST). Methods. 2016; 97: 27-34.

8. Duhr S, Braun D. Why molecules move along a 
temperature gradient. PNAS. 2006; 103(52): 19678-82.

9. Seidel SA, Dijkman PM, Lea WA, van den Bogaart G, Jerabek-Willemsen M, Lazic A, et al. Microscale thermophoresis quantifies biomolecular interactions under previously challenging conditions. Methods. 2013; 59(3): 301-15.

10. Wienken CJ, Baaske P, Rothbauer U, Braun D, Duhr S. Protein-binding assays in biological liquids using microscale thermophoresis. Nat Commun. 2010; 1: 100. doi: $10.1038 /$ ncomms 1093 .

11. Xiong X, Coombs PJ, Martin SR, Liu J, Xiao H, McCauley JW, et al. Receptor binding by a ferrettransmissible H5 avian influenza virus. Nature. 2013; 497(7449): 392-6.

12. van den Bogaart G, Meyenberg K, Diederichsen U, Jahn R. Phosphatidylinositol 4, 5-bisphosphate increases $\mathrm{Ca} 2+$ affinity of synaptotagmin- 1 by 40 -fold. J Biol Chem. 2012; 287(20): 16447-53.

13. Jerabek-Willemsen $M$, Wienken CJ, Braun $D$, Baaske P, Duhr S. Molecular interaction studies using microscale thermophoresis. Assay Drug Dev Technol. 2011; 9(4): 342-53.

14. Mao Y, Yu L, Yang R, Qu L-b, Harrington PdB. A novel method for the study of molecular interaction by using microscale thermophoresis. Talanta. 2015; 132: 894-901.

15. Asmari M, Ratih R, Alhazmi HA, El Deeb S. Thermophoresis for characterizing biomolecular interaction. Methods. 2018; 146: 107-19.

16. Bartoschik T, Maschberger M, Feoli A, André T, Baaske P, Duhr S, et al. Microscale thermophoresis in drug discovery. Applied Biophysics for Drug Discovery. 2017: p. 73-99.

17. Asadollahi E, Asadi A, Najafi F, Zahri S, Nasr SMH.
Biological properties of pegylated PLA (PLA-PEGPLA) and its capability for intracellular delivery of poor soluble peptide drug, gramicidin. International Journal of Drug Delivery. 2012; 4(2): 257-65.

18. Van Den Bogaart G, Thutupalli S, Risselada JH, Meyenberg K, Holt M, Riedel D, et al. Synaptotagmin-1 may be a distance regulator acting upstream of SNARE nucleation. Nat Struct Mol Biol. 2011; 18(7): 805-12.

19. Pobbati AV, Stein A, Fasshauer D. N-to C-terminal SNARE complex assembly promotes rapid membrane fusion. Science. 2006; 313(5787): 673-6.

20. Savolainen MH, Yan X, Myöhänen TT, Huttunen HJ. Prolyl oligopeptidase enhances $\alpha$-synuclein dimerization via direct protein-protein interaction. J Biol Chem. 2015; 290(8): 5117-26.

21. Wolff M, Mittag JJ, Herling TW, De Genst E, Dobson CM, Knowles TP, et al. Quantitative thermophoretic study of disease-related protein aggregates. Scientific Reports. 2016; 6: 22829.

22. Magnez R, Thiroux B, Taront S, Segaoula Z, Quesnel B, Thuru X. PD-1/PD-L1 binding studies using microscale thermophoresis. Scientific Reports. 2017; 7(1): 17623 .

23. NT020 AN, Breitsprecher D. Protein-DNA interaction analysis, dissection of complex interaction mechanisms by binding mode dependent thermophoresis signals ssDNA binding to EcoSSB. NanoTemper Technologies.

24. Topf A, Franz P, Tsiavaliaris G. MicroScale thermophoresis (MST) for studying actin polymerization kinetics. Biotechniques. 2017; 63(4): 187-90.

25. Jerabek-Willemsen M, André T, Wanner R, Roth HM, Duhr S, Baaske P, et al. MicroScale thermophoresis: interaction analysis and beyond. J Mol Struct. 2014; 1077: 101-13. 